Check for updates

Cite this: Nanoscale Adv., 2019, 1, 4947

\title{
Zirconia nanocomposites with carbon and iron(III) oxide for voltammetric detection of sub- nanomolar levels of methyl parathion $\uparrow$
}

\begin{abstract}
Krishna Prasad Gannavarapu, ${ }^{a}$ V. Ganesh ${ }^{\mathrm{b}}$ and Rajesh Babu Dandamudi (ID *a
This study reports the synthesis of zirconia nanoparticles loaded on various carbon substrates, namely, reduced graphene oxide ( $\mathrm{Zr}-\mathrm{r}-\mathrm{GO})$, carbon nanotubes ( $\mathrm{Zr}-\mathrm{CNT})$, and activated carbon ( $\mathrm{Zr}-\mathrm{AC})$. In addition, a composite of zirconia-iron mixed oxide loaded on activated carbon (FeZr-AC) was also synthesized. The materials were characterized using SEM-EDX, HRTEM, FTIR, Raman spectroscopy, TGA and XRD. The FeZr-AC sample was found to have a nanorod like morphology. The samples were evaluated for their sensing potential towards methyl parathion (MP) using differential pulse voltammetry in a range of $0.0 \mathrm{~V}$ to $-0.9 \mathrm{~V}(\mathrm{vs}$. $\mathrm{Ag} / \mathrm{AgCl}$ ) by drop casting on a glassy carbon electrode (GCE). All the modified GCEs best operated at a working potential of $0.4-0.9 \vee v s$. $\mathrm{Ag} / \mathrm{AgCl}^{-} \mathrm{Cl}^{-}$. FeZr-AC was found have the best limit of detection followed by $\mathrm{Zr}-\mathrm{AC}, \mathrm{Zr}$-CNT and $\mathrm{Zr}-\mathrm{r}-\mathrm{GO}$ with their detection limits being $1.7 \times 10^{-9} \mathrm{M}, 17.2 \times 10^{-9} \mathrm{M}, 243.3 \times 10^{-9} \mathrm{M}$ and $534.0 \times 10^{-9} \mathrm{M}$ respectively. These materials were then used to detect MP in spiked sewage samples and showed good recoveries.
\end{abstract}

Received 18th September 2019

Accepted 28th October 2019

DOI: $10.1039 / \mathrm{c} 9 \mathrm{na00589g}$

rsc.li/nanoscale-advances

selective, accurate and most importantly cheap. ${ }^{11}$ Many such sensors have been formulated to monitor and detect the levels of various OP pesticides. However, most of these methods essentially use enzyme-based techniques relying on the use of AChE and its inhibition activity to measure OP pesticides. ${ }^{12,13}$ These methods have shown acceptable low detection limits but suffer from the disadvantages of having higher costs and requiring careful handling..$^{12,14}$

The presence of the nitro group in MP makes it electrochemically active and hence can be directly detected using electrochemical methods. There have been many reports, which make use of nanoparticles as part of the working electrode to directly detect MP. These include gold, ${ }^{15,16}$ silver, zinc oxide, ${ }^{1,17}$ zirconia nanoparticles ${ }^{18-20}$ and other metal oxides. ${ }^{21,22}$ Among all of these nanoparticles, the use of zirconia is of particular interest due to its affinity towards the phosphate group present in MP. These nanoparticles themselves have a large surface area and by virtue of their affinity towards the phosphate group, offer a high level of sensitivity and selectivity. There have been no reports on the sensitivity and development of zirconia loaded on CNTs and activated carbon and a single report exists on zirconia nanoparticle loaded graphene for OP sensing. ${ }^{23}$ The loading of the zirconia nanoparticles on different substrates is of interest because the association with different substrates might lead to better sensitivity of the working electrode due to varied conductivity and surface area of the substrates.

In the current work, we report a facile microwave mediated synthesis of zirconia nanoparticles loaded on graphene (Zr-r$\mathrm{GO}$ ), CNTs (Zr-CNT) and activated carbon (Zr-AC) derived from a spent mushroom substrate. In addition, we have also 
synthesized iron oxide-zirconia nanoparticles on AC (FeZr-AC) and studied their activity in the electrochemical sensing of MP. All the composites were characterized using SEM-EDX, HRTEM, FTIR, Raman spectroscopy, TGA and XRD. A lowest detection limit of $1.7 \times 10^{-9} \mathrm{M}$ was achieved for Fe-Zr-AC followed by $\mathrm{Zr}$ AC, Zr-CNT and Zr-r-GO. All the composites sensed MP over a large range of linearity. The selectivity of the composites was retained even in the presence of interfering molecules and ions at high concentrations.

\section{Materials and methods}

\section{Materials}

The sawdust used for the preparation of the activated carbon was obtained from the mushroom culture facility in the department. Phosphoric acid, potassium hydroxide, ferrous sulphate, sodium hydroxide, sodium dihydrogen orthophosphate, disodium hydrogen orthophosphate, graphite, potassium hexacyanoferrate(III) trihydrate, potassium hexacyanoferrate(II), hydrogen peroxide, hydrazine hydrate, potassium hydroxide, nitric acid and sulphuric acid were of analytical grade and were obtained from Merck (http:// www.merckmillipore.com). The MP standard was purchased from Sigma-Aldrich. All the solutions were prepared in Milli-Q water (resistivity $=18.2 \mathrm{M} \Omega \mathrm{cm}$ at $25^{\circ} \mathrm{C}$ ).

\section{Instruments}

The SEM and EDX data were obtained on a Jeol IT300 by putting a small amount of sample on carbon tape. The HRTEM images were obtained on a Jeol 2100 Plus instrument by drop casting the dispersed sample onto a copper grid. TGA was performed on a TA SDTQ600 by taking $5 \mathrm{mg}$ of the sample in an alumina pan and heating until $800{ }^{\circ} \mathrm{C}$ at a rate of $10{ }^{\circ} \mathrm{C} \min ^{-1}$ under a nitrogen flow. Microwave reduction was carried out using a Mars 6 (CEM). Raman spectra were obtained using a Thermo Scientific DSR Raman microscope equipped with a $780 \mathrm{~nm}$ laser. FTIR spectra were recorded on an Agilent Cary 630 FTIR spectrometer in ATR mode. Powder XRD patterns were recorded on a Panalytical Xpert-3. GC-MS analysis was carried out on a Shimadzu QP 2010 SE equipped with an AOC $20 \mathrm{i}$ autosampler.

\section{Methods}

\section{Synthesis of activated carbon}

The sawdust precursor ${ }^{24}$ received from the mushroom cultivation facility of the department was washed with hot water, filtered and dried at $120{ }^{\circ} \mathrm{C}$. The dried material was taken in a round bottom flask and charred with $60 \%$ ortho-phosphoric acid by heating it up to $150{ }^{\circ} \mathrm{C}$. The obtained char was annealed at $500{ }^{\circ} \mathrm{C}$ in a tube furnace at a ramp rate of $5{ }^{\circ} \mathrm{C} \mathrm{min}{ }^{-1}$ under a flow of nitrogen at $100 \mathrm{~mL} \mathrm{~min}^{-1}$. Thus, the obtained product was washed with copious amounts of distilled water and ethanol until neutral $\mathrm{pH}$, dried at $70{ }^{\circ} \mathrm{C}$ in a vacuum oven overnight and labelled SDAC500.

SDAC500 was mixed with a solution of $3 \mathrm{M} \mathrm{KOH}$ such that the SDAC500/KOH ratio is $1: 3$. The dry mixture was annealed at $800{ }^{\circ} \mathrm{C}$ in a tube furnace, at a ramp rate of $5{ }^{\circ} \mathrm{C}$ min under

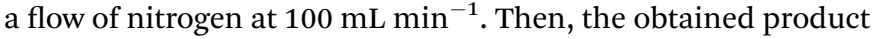
was washed with copious amounts of distilled water and ethanol, dried at $70{ }^{\circ} \mathrm{C}$ in a vacuum oven overnight and labelled SDAC800. ${ }^{25}$

\section{Synthesis of graphene oxide (GO)}

GO was prepared using the well-known Hummers process. ${ }^{26}$ In a typical experiment, $1 \mathrm{~g}$ of graphite powder along with $0.5 \mathrm{~g}$ of sodium nitrate was added to $23 \mathrm{~mL}$ of $98 \%$ sulphuric acid and cooled to $0{ }^{\circ} \mathrm{C}$ in an ice bath. To this mixture, $30 \mathrm{~g}$ of potassium permanganate was added with vigorous stirring ensuring that the temperature did not increase above $20^{\circ} \mathrm{C}$. This mixture was maintained at $35{ }^{\circ} \mathrm{C}$ for $30 \mathrm{~min}$. The progress of the reaction was indicated by the formation of a brown paste with decrease in effervescence at the end of $20 \mathrm{~min}$. At the end of $30 \mathrm{~min}, 46 \mathrm{~mL}$ of water was carefully added to the reaction mixture, which resulted in violent effervescence along with an increase in temperature to $98{ }^{\circ} \mathrm{C}$. The reaction mixture was maintained at this temperature for $15 \mathrm{~min}$. Later, the reaction mixture was further diluted with $140 \mathrm{~mL}$ of water and treated with hydrogen peroxide, which resulted in the mixture turning yellow. This was filtered while hot and washed with $70 \%$ ethanol and water for two cycles. The precipitate was then re-dispersed in $320 \mathrm{~mL}$ of water and dialyzed until the total dissolved salt content was constant in water. Finally, the residue was centrifuged at 10000 rpm and the resultant precipitate was dried in a vacuum.

\section{Functionalization of SDAC800 and mwCNTs}

mwCNTs ( 0.5 to $1.0 \mu \mathrm{m}$ in length with their OD and ID being 70 $\mathrm{nm}$ and $20 \mathrm{~nm}$ respectively) and SDAC500 were added to concentrated $\mathrm{HNO}_{3}$ in a $10: 1 \mathrm{wt} / \mathrm{vol}$ ratio and microwave irradiated with 1000 watts power in a Mars CEM EasyPrep vessel at $100{ }^{\circ} \mathrm{C}$ for $30 \mathrm{~min}$. The reaction mixture was then cooled down to room temperature and filtered under vacuum using a 0.22 $\mu \mathrm{m}$ PTFE membrane filter. The product was then washed thoroughly with water (Milli-Q) and dried in a vacuum oven overnight. The products were collected and labeled f-mwCNT and f-SDAC800.

\section{Synthesis of $\mathrm{ZrO}_{2}$ nanoparticle loaded r-GO, CNT and SDAC800}

$\mathrm{ZrO}_{2}$ loaded r-GO. $100 \mathrm{mg}$ of the synthesized GO above was dispersed in $50 \mathrm{~mL}$ of Milli-Q water by ultra-sonication to which $50 \mathrm{~mL}$ of $10 \mathrm{mM} \mathrm{ZrOCl}{ }_{2}$ solution was added and sonicated for $20 \mathrm{~min}$. To this well-dispersed solution, $1 \mathrm{~mL}$ of hydrazine hydrate was added and the mixture was microwave irradiated with 1000 watts power in a Mars CEM EasyPrep vessel at $150{ }^{\circ} \mathrm{C}$ for $40 \mathrm{~min}$. The obtained product was filtered under vacuum using $0.22 \mu \mathrm{m}$ PTFE membrane filters, washed thoroughly with water (Milli-Q) and dried in a vacuum overnight. The product was weighed and labelled Zr-r-GO.

$\mathrm{ZrO}_{2}$ loaded mwCNT/SDAC800. f-mwCNTs $(300 \mathrm{mg}$ ) and fSDAC800 (300 mg) were dispersed in $100 \mathrm{~mL}$ of $10 \mathrm{mM} \mathrm{ZrOCl}$ solution and sonicated for $20 \mathrm{~min}$. This well-dispersed solution was transferred to a Mars CEM EasyPrep vessel and microwave 
irradiated with 1000 watts power at $150{ }^{\circ} \mathrm{C}$ for $40 \mathrm{~min}$. The obtained product was filtered under vacuum using $0.22 \mu \mathrm{m}$ PTFE membrane filters, washed thoroughly with water (Milli-Q) and dried in a vacuum overnight. The product was weighed and labelled Zr-CNT and Zr-AC respectively.

$\mathrm{FeO}_{x}-\mathrm{ZrO}_{2}$ nanorods loaded on SDAC800. $500 \mathrm{mg}$ of SDAC800 was well dispersed in $200 \mathrm{~mL}$ of nitrogen purged Milli$\mathrm{Q}$ water by ultra-sonication. To this solution, $700 \mathrm{mg}$ of $\mathrm{FeSO}_{4} \cdot 7 \mathrm{H}_{2} \mathrm{O}$ and $1.5 \mathrm{~mL}$ of $0.25 \mathrm{mM} \mathrm{ZrOCl}_{2}$ solution were added and heated to $60{ }^{\circ} \mathrm{C}$ in a microwave reactor initially. The $\mathrm{pH}$ of this solution was adjusted to 11 using $3 \mathrm{M} \mathrm{NaOH}$ solution and the resulting solution was transferred to a Mars CEM EasyPrep vessel and microwave irradiated with 1000 watts power at $150{ }^{\circ} \mathrm{C}$ for $20 \mathrm{~min}$. The obtained product was filtered under vacuum using $0.22 \mu \mathrm{m}$ PTFE membrane filters, washed thoroughly with water (Milli-Q) and dried in a vacuum overnight. The product was weighed and labeled FeZr-AC.

\section{Electrochemical measurements}

Electrochemical studies involving cyclic voltammetry and differential pulse voltammetry were carried out in a three electrode system setup on a Biologic VSP300, a potentiostat/galvanostat system. The working electrode used was a glassy carbon electrode (GCE) which was polished using 0.3 and $0.05 \mu \mathrm{m}$ alumina slurry and was further cleaned by sonicating in water and ethanol. The counter electrode used was a platinum wire cleaned using dilute HCL and the reference electrode used was a $\mathrm{Ag} / \mathrm{AgCl} / \mathrm{Cl}^{-}$electrode. The main working electrode for the sensing studies was fabricated by drop casting $10 \mu \mathrm{l}$ of the slurry of the electrode materials pre-dispersed $\left(1 \mathrm{mg} \mathrm{ml}^{-1}\right)$ in Milli-Q water and by drying the same in a vacuum.

\section{Results and discussion}

\section{Characterization}

The surface morphology of the samples was evaluated using SEM (Fig. S1†) and HRTEM. The HRTEM images showed the presence of $\mathrm{ZrO}_{2}$ nanoparticles (Fig. 1) in all samples. In the case of FeZr-AC, the particles can be seen in the form of nanorods (Fig. 2a and b). The EDX spectra of the samples were recorded to estimate the percentage loading of zirconia on various substrates. The results are summarized in Table 1 . The EDX data indicated the presence of $\mathrm{ZrO}_{2}$ in the samples. Moreover, to confirm the homogeneous presence of the nanoparticles in all samples, XPS mapping was carried out at $184 \mathrm{eV}$ for $\mathrm{Zr} 3 \mathrm{~d}_{3 / 2}, 287 \mathrm{eV}$ for $\mathrm{C} 1 \mathrm{~s}, 530 \mathrm{eV}$ for $\mathrm{O} 1 \mathrm{~s}$ and $730 \mathrm{eV}$ for $\mathrm{Fe}$ $2 \mathrm{p}_{1 / 2}$ (Fig. $\mathrm{S} 2 \dagger$ ). The images clearly indicated the uniform presence of $\mathrm{Zr}$ in the composites $\mathrm{Zr}$-r-Go, Zr-CNT and Zr-AC along with Fe in FeZr-AC.

The FTIR spectra showed the presence of the $-\mathrm{COOH}$ functionality in samples SDAC800 and mwCNT (Fig. S4 $\dagger$ ) as indicated by the increase in the intensities of $\nu_{-\mathrm{OH}}$ at $3400 \mathrm{~cm}^{-1}$ and $\nu_{-\mathrm{C}=\mathrm{O}}$ at around $1610 \mathrm{~cm}^{-1}$ from SDAC800 and mwCNT to $\mathrm{f}-$ SDAC800 and f-mwCNT respectively. The FTIR spectra (Fig. S3a$\mathrm{c} \dagger$ ) also indicated the formation of zirconia particles on various substrates. The small absorption peak at $470 \mathrm{~cm}^{-1}$ along with the peaks at $613 \mathrm{~cm}^{-1}$ and $819 \mathrm{~cm}^{-1}$ for $\mathrm{Zr}-\mathrm{O}-\mathrm{Zr}$ vibrations is indicative of $\mathrm{ZrO}_{2}$ particle formation. ${ }^{27} \mathrm{Zr}-\mathrm{AC}$, Zr-CNT and $\mathrm{Zr}-\mathrm{r}$ GO have strong absorption bands at around $1700 \mathrm{~cm}^{-1}$ indicating the presence of $\nu_{-\mathrm{C}=\mathrm{O}}$, a reminiscent vibration due to functionalization to anchor the $\mathrm{ZrO}_{2}$ particles. However, this peak is absent in the FeZr-AC material as the activated carbon substrate here was not functionalized. The sharp peak at 3000
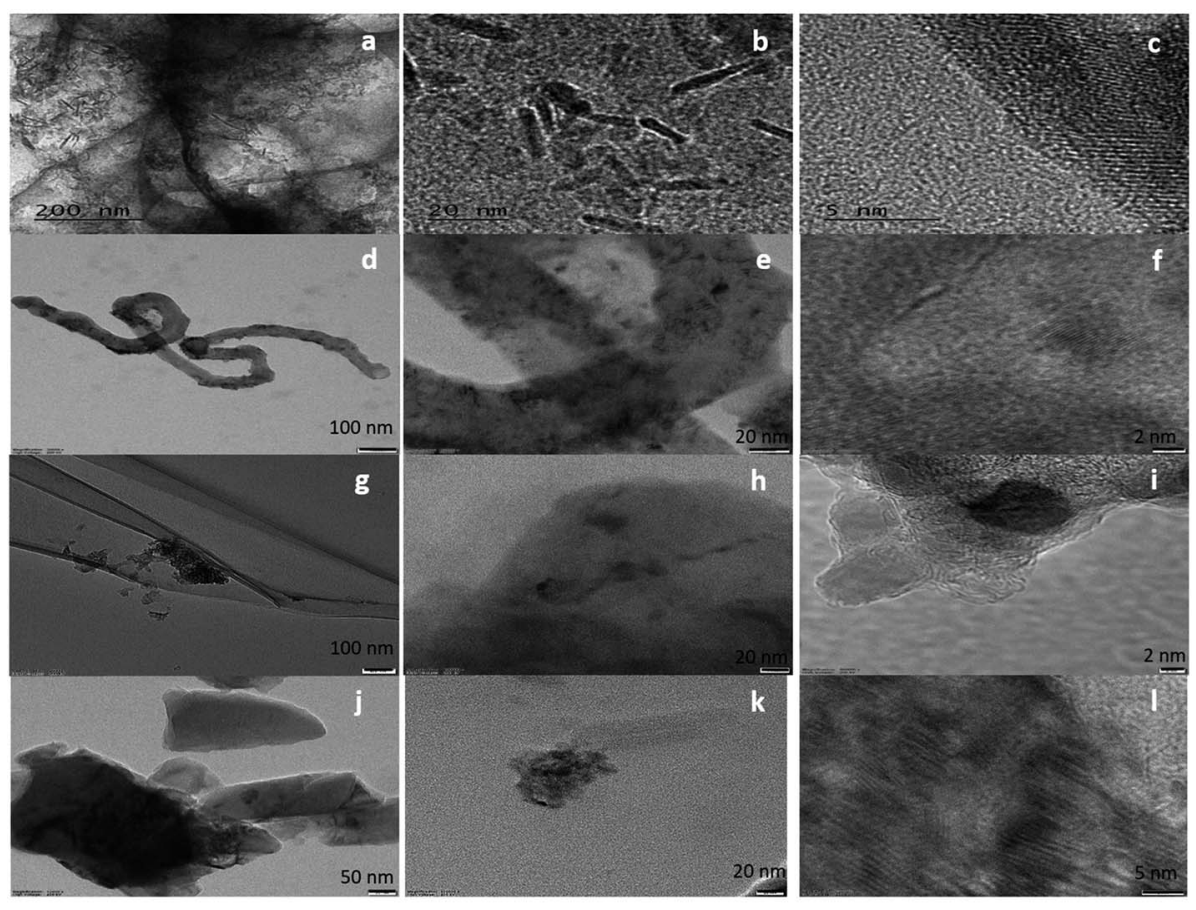

Fig. 1 HRTEM images of (a-c) FeZr-AC, (d-f) $\mathrm{ZrO}_{2}-\mathrm{CNT}$, (g-i) $\mathrm{ZrO}_{2}-\mathrm{r}-\mathrm{GO}$ and (j-l) $\mathrm{ZrO}_{2}-\mathrm{AC}$ at different scales. 

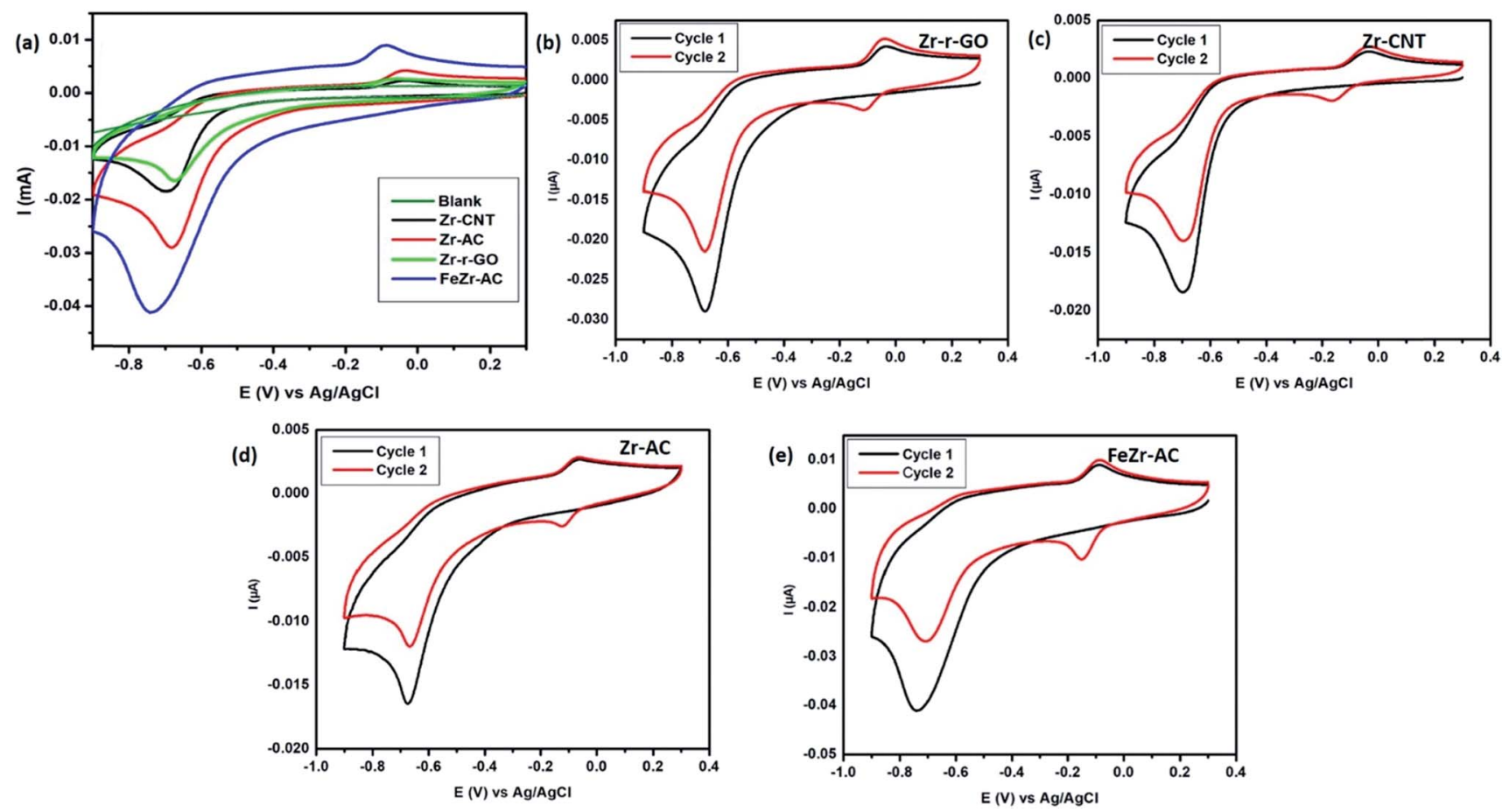

Fig. 2 Cyclic voltammograms of the different materials viz., Zr-r-GO, Zr-CNT, Zr-AC and FeZr-AC $2.5 \mu \mathrm{gl}^{-1}$ of MP (15 mM PB, pH 7.2) at a scan rate of $50 \mathrm{mV} \mathrm{s}^{-1}$. (a) Comparison of the materials; (b)-(e) cyclic voltammograms of the materials for cycle 1 and 2 to understand the mechanism. The CVs were recorded by sweeping from positive potential to negative potential from $0.3 \mathrm{~V}$ to $-0.9 \mathrm{~V}$.

Table 1 Loading of $\mathrm{Zr}$ on different substrates as obtained from EDX spectra

\begin{tabular}{ll}
\hline Sample & Loading (atomic \%) \\
\hline $\mathrm{ZrO}_{2}-\mathrm{r}-\mathrm{GO}$ & 3.84 \\
$\mathrm{ZrO}_{2}-\mathrm{CNT}$ & 3.19 \\
$\mathrm{ZrO}_{2} / \mathrm{AC}$ & 3.24 \\
$\mathrm{FeZr}-\mathrm{AC}(\mathrm{Fe}, \mathrm{Zr})$ & $3.89(\mathrm{Fe}), 2.22(\mathrm{Zr})$
\end{tabular}

$\mathrm{cm}^{-1}$ in $\mathrm{Zr}-\mathrm{r}-\mathrm{GO}$ is due to the symmetric stretching of unsaturated $-\mathrm{C}-\mathrm{H}$ bonds. The common peaks for all the samples were at $3500 \mathrm{~cm}^{-1}, 1550 \mathrm{~cm}^{-1}$ and $1100 \mathrm{~cm}^{-1}$ and can be attributed to the $\nu_{-\mathrm{OH}}$ symmetric stretching, skeletal $\mathrm{C}=\mathrm{C}$ stretching and C-O stretching respectively.

The Raman spectra (Fig. S3d-g $\dagger$ ) of the samples indicated the graphitic nature of the substrates (Fig. S5 $\dagger$ ). The peaks at $150 \mathrm{~cm}^{-1}, 300 \mathrm{~cm}^{-1}, 450 \mathrm{~cm}^{-1}$, and $650 \mathrm{~cm}^{-1}$ correspond to zirconia (shown in the insets) in its tetragonal form. ${ }^{28,29}$ The presence of these peaks clearly states the formation of $\mathrm{ZrO}_{2}$ nanoparticles. In the case of FeZr-AC, the peaks at $255 \mathrm{~cm}^{-1}$ (shoulder), $299 \mathrm{~cm}^{-1}$ and $613 \mathrm{~cm}^{-1}$ correspond to $\alpha-\mathrm{Fe}_{2} \mathrm{O}_{3}$. These peaks originate from $\mathrm{E}_{\mathrm{g}}$ modes of $\alpha-\mathrm{Fe}_{2} \mathrm{O}_{3}$. The decrease in the intensity of the G-band along with the increase of the same in the D-band when compared to the substrates clearly indicates the presence of zirconia nanoparticles on the substrates (Table $\mathrm{S} 1 \dagger$ ).

The XRD spectra (Fig. S6 $\dagger$ ) also support the conclusions drawn from the other techniques. The $2 \theta$ peaks at $30.20,35.28$ and 50.76 correspond to the (101), (110) and (200) planes of
$\mathrm{ZrO}_{2}$ in the tetragonal phase concurring with the Raman spectra. The FeZr-AC sample has shown $2 \theta$ peaks at 24.12, 33.11 and 63.96 which correspond to the (012), (104) and (300) planes of $\alpha-\mathrm{Fe}_{2} \mathrm{O}_{3}$, strengthening the findings from Raman spectra. The peaks observed at $2 \theta$ values of 26.21 and 43.56 corresponding to the (002) and (100) lattice planes of carbon were also seen.

\section{Electrochemical studies}

Cyclic voltammetry was employed in order to study the electrochemical response of different electrode materials towards MP. The cyclic voltammograms are shown in Fig. 2a. The reduction of $\mathrm{MP}$ occurs at $-0.67 \mathrm{~V},-0.69 \mathrm{~V},-0.68 \mathrm{~V}$ and -0.73 $\mathrm{V}$ for Zr-r-GO, Zr-CNT, Zr-AC and FeZr-AC loaded GCE, respectively, while no reduction current for MP was observed with the plain GCE. It is also interesting to note that for the same amount of MP, the reduction peak current was maximum for FeZr-AC followed by Zr-AC, Zr-CNT and Zr-r-GO respectively. This may be attributed to the higher surface area of the materials in FeZr-AC and $\mathrm{Zr}-\mathrm{AC}$. Moreover, the improved performance of FeZr-AC may be due to improved conductance because of synergistic effects between $\mathrm{Fe}$ and $\mathrm{Zr}$ in the composite. The increased surface area also helps in better adsorption of MP leading to higher reduction currents.

\section{Electroanalytical studies}

Differential pulse voltammetry (DPV) experiments were designed and carried out to understand and develop these materials as possible non-enzymatic analytical tools for the detection of MP. All the DPV experiments were carried out by 
sweeping the potential from $0 \mathrm{~V}$ to $-0.9 \mathrm{~V}$ in $15 \mathrm{mM} \mathrm{PB}$ with $\mathrm{pH}$ 7.2 as the supporting electrolyte. The pulse height and pulse width for the experiment were standardized to be $50 \mathrm{mV}$ with a step height of $-5.0 \mathrm{~V}$. The differential pulse voltammograms (Fig. 3) indicate that for all the studied materials, the reduction current increases systematically with increase in the (a)
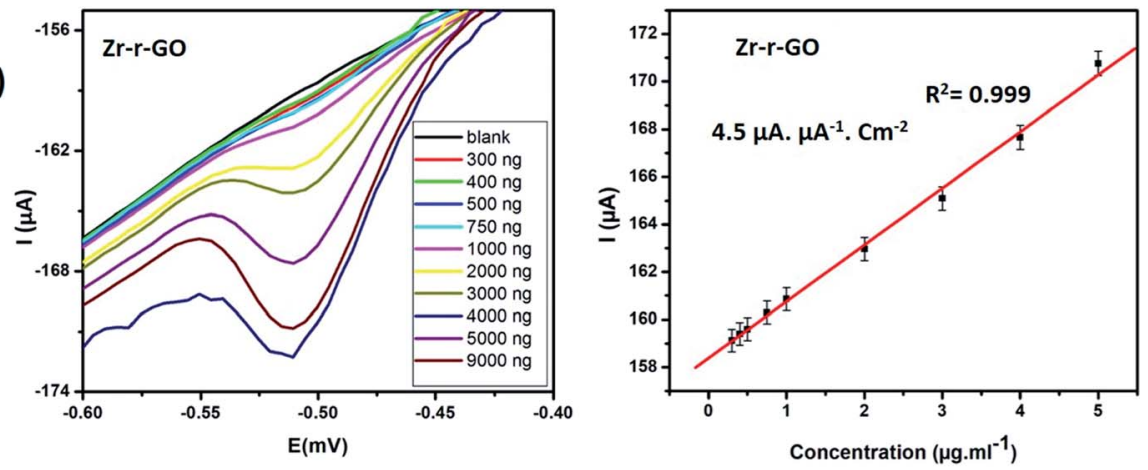

(b)
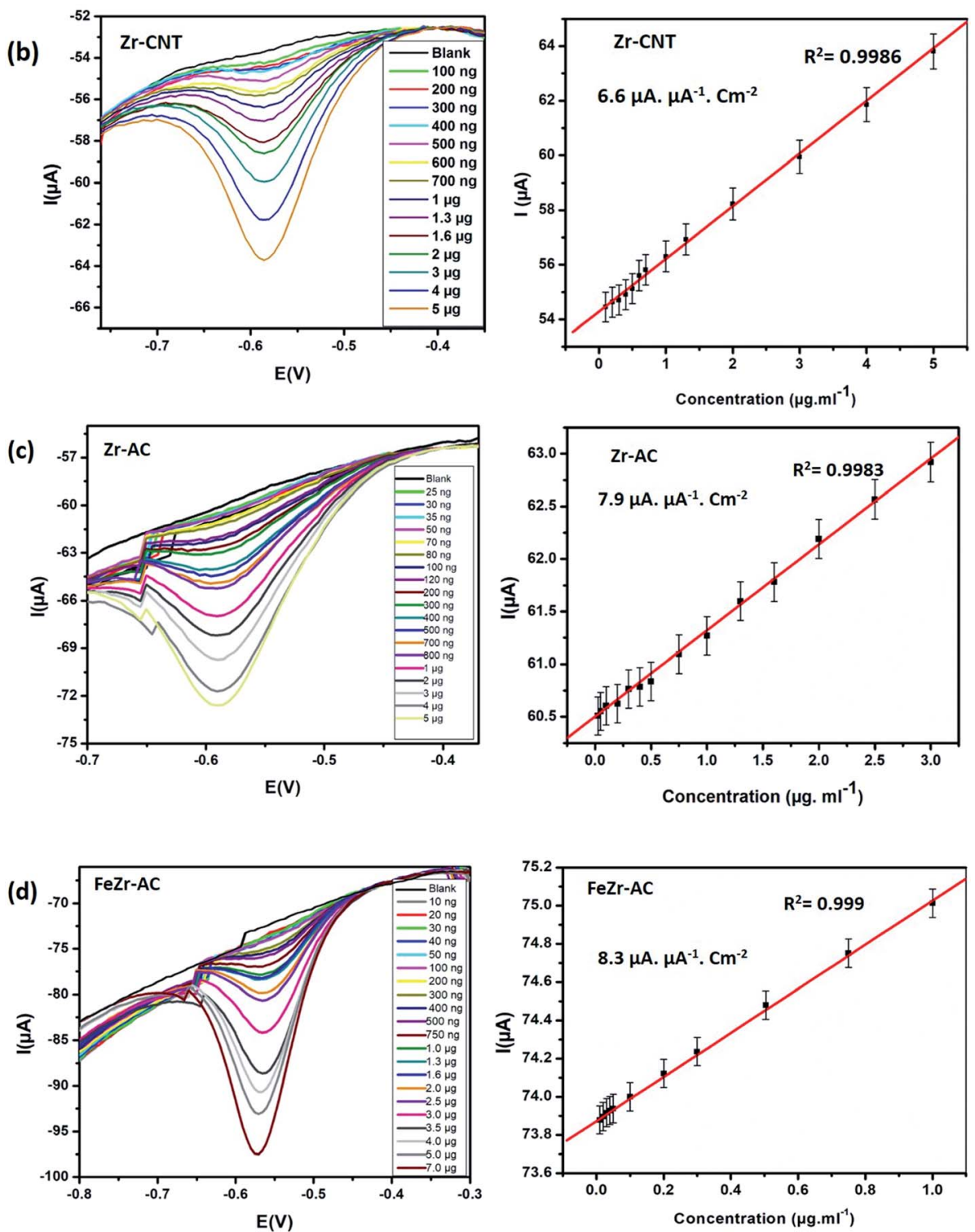

Fig. 3 DPV voltammograms and the corresponding linearity plots obtained at increasing concentrations for (a) Zr-r-GO, (b) Zr-CNT, (c) Zr-AC and (d) FeZr-AC. 
Table 2 Limit of detection and linearity range of samples $\mathrm{Zr}$-r-GO, $\mathrm{Zr}$-CNT, $\mathrm{Zr}$-AC and FeZr-AC and comparison with other electrode materials from the literature

\begin{tabular}{|c|c|c|c|}
\hline Electrode material/type of electrode & Limit of detection (mol) & Linear concentration-range (mol) & Reference \\
\hline Zr-r-GO/GCE & $534.0 \times 10^{-9}$ & $1.0 \times 10^{-6}$ to $30.9 \times 10^{-6}$ & This work \\
\hline Zr-CNT/GCE & $243.3 \times 10^{-9}$ & $0.3 \times 10^{-6}$ to $34.3 \times 10^{-6}$ & This work \\
\hline FeZr-AC/GCE & $1.7 \times 10^{-9}$ & $34.4 \times 10^{-9}$ to $68.7 \times 10^{-6}$ & This work \\
\hline $\mathrm{ZrO}_{2}-\mathrm{Au}$ & $10.3 \times 10^{-9}$ & $17.2 \times 10^{-9}$ to $0.3 \times 10^{-6}$ & 30 \\
\hline CNT/carbon paper & $10.3 \times 10^{-9}$ & $34.3 \times 10^{-9}$ to $3.4 \times 10^{-6}$ & 31 \\
\hline Mesoporous carbon/GCE & $7.6 \times 10^{-9}$ & $90 \times 10^{-9}$ to $61 \times 10^{-6}$ & 33 \\
\hline Silicate/GCE & $10 \times 10^{-9}$ & $1.0 \times 10^{-7}$ to $1.0 \times 10^{-4}$ & 34 \\
\hline GR-CS/GCE & $2.7 \times 10^{-9}$ & $13.7 \times 10^{-9}$ to $1.37 \times 10^{-6}$ & 35 \\
\hline
\end{tabular}

concentration of MP. A minor shift in the reduction peak potential was observed to the negative side and this is explained by the possible increase in the resistance of the solution with increase in the concentration of MP. This shift is generally between 10 and $20 \mathrm{mV}$ and the same has been noticed for all the materials. From Fig. 3, it is clear that all the materials maintain a linear range of detection across low to higher concentrations and the data for the same are summarized in Table 2.

Of the studied materials Zr-Ac and FeZr-AC showed particularly lower detection limits and exhibited linearity over a wider range of concentrations. The better activity of the materials derived from activated carbon can be attributed to their higher surface area and possible better conductivity. The better performance of FeZr-AC is attributed to the synergistic effect between $\mathrm{Fe}$ and $\mathrm{Zr}$. To study this, we have recorded and compared the cyclic voltammograms of Zr-AC, FeZr-AC and FeAC (Fig. S9†). We observed that the material Fe-AC showed capacitive behavior when compared to FeZr-AC and Zr-AC. This is expected as iron is in the oxide form. We also found that it showed a weak signal for the analyte, MP, and so may not be a suitable sensor material.

\section{Interference studies}

Selectivity plays a major role in determining the worth of any electrode material as a viable sensor. In order to ascertain the selectivity of the electrode materials towards MP without any ubiquity, DPV experiments were carried out in the presence of some of the commonly studied interfering ions and nitro aromatic compounds. The analysis was carried out in the presence of nitrobenzene (NB), $p$-nitrophenol (PNP) and $p$ nitroaniline (NA) in ten times higher concentration for each when compared to MP. A thousand-fold higher quantities of $\mathrm{Na}^{+}, \mathrm{K}^{+}, \mathrm{Ca}^{2+}, \mathrm{CO}_{3}{ }^{2-}, \mathrm{PO}_{4}{ }^{2-}, \mathrm{Cl}^{-}, \mathrm{Fe}^{2+} / \mathrm{Fe}^{3+}, \mathrm{NO}_{3}{ }^{-}$and $\mathrm{NO}_{2}{ }^{-}$ were used for interference studies. The amount of MP was kept constant at $2.5 \mu \mathrm{g} \mathrm{ml} \mathrm{m}^{-1}$ and the results are summarized in Fig. 4. It was noticed that the interference current was less than $5 \%$ in the case of FeZr-AC and the highest of $8 \%$ in the case of Zr-r-GO, making a strong statement in favor of the high selectivity of the materials to MP. The higher interference in the case of Zr-r-GO can be expected due to possible $\pi-\pi$ interactions with the aromatic compounds, which is not possible in the case of the AC substrate.

\section{Mechanism of sensing of MP}

It is important to understand the mechanism of sensing MP in order to fabricate better sensors. An attempt has been made to elucidate the mechanism of electrochemical signal generation by MP with the help of cyclic voltammograms obtained (Fig. 2be). This has been thoroughly discussed in the ESI. $\dagger^{\mathbf{1 8 , 3 6}}$ The CVs recorded without MP yielded no reduction peaks, asserting that there is no redox reaction happening. In addition, the reduction process occurring in the first step was studied at different scan rates of $10,20,30,40,60,80$ and $100 \mathrm{mV} \mathrm{s}^{-1}$ (Fig. S9†) to determine whether the process is diffusion controlled or surface confined. It was noticed that there was a steady increase in the reduction current with an increase in the scan rate and the slopes of the logarithmic plots of the corresponding peak current $v s$. scan rate were calculated to be $0.64,0.71,0.68$ and 0.77 for Zr-r-GO, Zr-CNT, Zr-AC and FeZr-AC respectively. It is known that if this slope equals to 0.5 then the corresponding redox process is only diffusion controlled and if the value equals

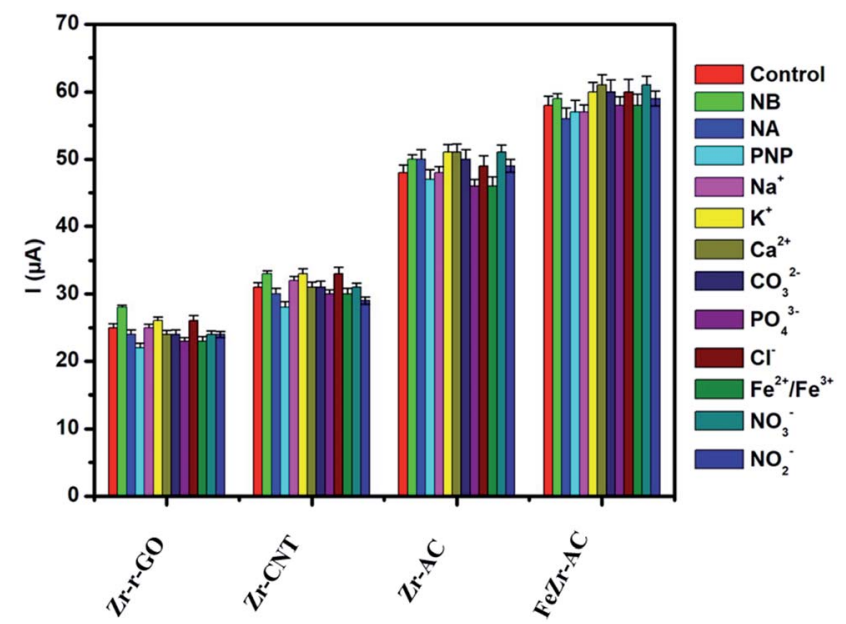

Fig. 4 Interference studies performed on the materials $\mathrm{Zr}$-r-GO, $\mathrm{Zr}$ CNT, $\mathrm{Zr}-\mathrm{AC}$ and FeZr-AC in $2.5 \mu \mathrm{g} \mathrm{ml}^{-1}$ of MP (15 mM PB, pH 7.2). 
Table 3 Recovery studies for spiked sewage water along with the standard deviation where $n=3$

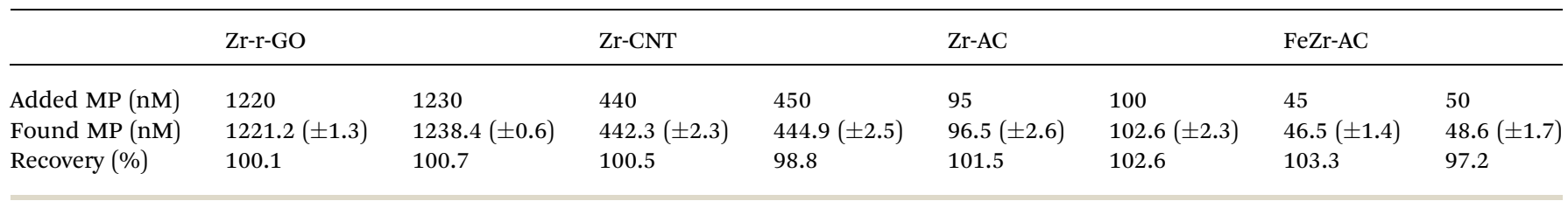

1.0 then the process is a surface confined process. ${ }^{37}$ The slope values indicate that the process is both surface confined and diffusion controlled.

The presence of the zirconia nanoparticles primarily has led to the selectivity and sensitivity of the studied materials. It has been previously studied and understood that the interaction of zirconia with phosphate occurs via the inner sphere mechanism with the $\mathrm{Zr}-\mathrm{O}$ bond interacting with $\mathrm{P}$. This is however absent in the case of other moieties like $\mathrm{SO}_{4}{ }^{2-}, \mathrm{NO}^{3-}$ and $\mathrm{Cl}^{-} .^{38} \mathrm{MP}$ has a thiophosphoryl group which interacts with the zirconia present in the electrode materials. This strong interaction is deemed responsible for endowing the composites with good sensitivity and selectivity. This hypothesis is further strengthened due to weaker signals obtained from analytes like $p$ nitrophenol (PNP), nitrobenzene (NB), and $p$-nitroaniline (NA) which lack any phosphorus moiety.

Moreover, it has been observed that the FeZr-AC composites exhibit better sensitivity when compared to $\mathrm{Zr}-\mathrm{AC}$, Zr-CNT and $\mathrm{Zr}$-r-GO composites. To further investigate this, the electrochemically active surface area (A) of these materials was calculated using the Randles-Sevcik equation and the conductance of the samples were calculated using the Nicholson equation by evaluating the electron rate transfer coefficient $\left(K_{\mathrm{obs}}^{\mathrm{O}}\right)$ for the simple ferri-ferro redox system. The data are summarized in Table S2. $\uparrow$ The results point out that the higher active surface area and better conductance of FeZr-AC help in achieving better sensitivity for the material when compared to others.

\section{Real sample analysis}

In order to demonstrate the feasibility of the samples to be used as MP sensors in real samples, sewage water from the university site (SSSIHL, Puttaparthi) was collected and the analysis was carried out. Initially the water sample was filtered through a 0.22 $\mu$ membrane filter to remove the particulates and was analyzed for the presence of MP. The analysis of this sample showed no response for MP indicating the absence of the analyte in the water. Recovery studies were carried out by spiking this water sample with known concentrations of MP. Two different concentrations were used for each electrode material and the results are summarized in Table 3. All the samples showed good recovery values and demonstrate the potential usage of these as electrode materials for detecting MP in environmental samples. The recovery rates obtained by the electrochemical method have been verified using the standard GC-MS technique and the results are presented in Table S3 of the ESI. $\dagger$

In real samples, parathions are known to slowly oxidize to paraoxons. The presence of these should also be detected if assessment with respect to toxic effects of MP needs to be carried out in total.

\section{Conclusions}

In the current work, we have demonstrated novel carbon based composites for selective and sensitive enzyme free electrochemical sensing for the detection of organo-phosphor class pesticide methyl parathions and have compared them for their sensitivity towards MP. A facile microwave mediated synthetic procedure was employed to synthesize zirconia loaded carbon substrates Zr-r-GO, Zr-CNT, Zr-AC and FeZr-AC which were characterized using SEM-EDX, HRTEM, FTIR, Raman spectroscopy, TGA and XRD. The FeZr-AC sample was found to have nanorod like morphology with the $\mathrm{Zr}$ loading being uniform in all the samples. The samples were evaluated for their sensing potential towards MP where FeZr-AC was found to be the most highly sensitive material followed by Zr-AC, Zr-CNT and Zr-r-GO respectively. It was also found that all the materials were highly selective towards MP even in the presence of other interfering molecules and ions, showing a high recovery percentage when spiked real samples were analyzed. We hope that these composite materials find application as disposable electrodes in MP detection. Further studies need to be conducted to detect MP in the presence of similar organophosphate pesticides.

\section{Conflicts of interest}

There are no conflicts to declare.

\section{Acknowledgements}

The authors dedicate the work to Bhagawan Sri Sathya Sai Baba, Founder Chancellor - Sri Sathya Sai Institute of Higher Learning. The authors also acknowledge Sri Sathya Sai Institute of Higher Learning-Central Research Instrument Facility (SSSIHL-CRIF) for characterization facilities and infrastructure. The authors recognize the support of the central library, SSSIHL, for providing access to Scopus. The authors are grateful to late. Prof. Panduranga Rao, Department of English, SSSIHL, for his due help in the preparation of the manuscript. The authors acknowledge the funding and support from DST-FIST (SR/FST/PSI-172/2012) and UGC, India, for purchase of the Raman microscope. The authors also acknowledge Dr V. Sai Muthu Kumar, Department of Physics, Sri Sathya Sai Institute of Higher Learning, for providing access to the Raman microscope during the course of this work. 


\section{References}

1 D. Sahoo, A. Mandal, T. Mitra, K. Chakraborty, M. Bardhan and A. K. Dasgupta, J. Agric. Food Chem., 2018, 66(2), 414423.

2 J. Yang, C. Yang, H. Jiang and C. Qiao, Biodegradation, 2008, 19, 831-839.

3 M. Nurdin, M. Maulidiyah, M. Z. Muzakkar and A. A. Umar, Microchem. J., 2019, 145, 756-761.

4 L. Gao, L. Ju and H. Cui, J. Mater. Chem. C, 2017, 5, 77537758.

5 G. Koukouvinos, Z. Tsialla, P. S. Petrou, K. Misiakos, D. Goustouridis, A. U. Moreno, A. R. Fernandez-Alba, I. Raptis and S. E. Kakabakos, Sens. Actuators, B, 2017, 238, 1214-1223.

6 P. Carullo, G. P. Cetrangolo, L. Mandrich, G. Manco and F. Febbraio, Sensors, 2015, 15, 3932-3951.

7 F. Salam, N. H. A. Puat, G. A. Rahman, S. N. M. Ramli, K. A. Mohamed, A. A. A. Kadir, A. Azmi, M. F. Nasaruddin, M. M. Saad and Z. Ishak, Procedia Chem., 2016, 20, 33-39.

8 J. Chen, W.-t. Zhang, Y. Shu, X.-h. Ma and X.-y. Cao, Food Analytical Methods, 2017, 10, 3452-3461.

9 G. Gauglitz, B. Wimmer, T. Melzer and C. Huhn, Anal. Bioanal. Chem., 2017, 1-22.

10 Y. Fan, L. Liu, D. Sun, H. Lan, H. Fu, T. Yang, Y. She and C. Ni, Anal. Chim. Acta, 2016, 916, 84-91.

11 A. J. Bandodkar and J. Wang, Trends Biotechnol., 2014, 32, 363-371.

12 N. Xia and Y. Gao, Int. J. Electrochem. Sci., 2015, 10, 713-724.

13 S. Viswanathan, H. Radecka and J. Radecki, Biosens. Bioelectron., 2009, 24, 2772-2777.

14 H. Parham and N. Rahbar, J. Hazard. Mater., 2010, 177, 1077-1084.

15 Y. Zhang, T.-F. Kang, Y.-W. Wan and S.-Y. Chen, Microchim. Acta, 2009, 165, 307-311.

16 T.-F. Kang, F. Wang, L.-P. Lu, Y. Zhang and T.-S. Liu, Sens. Actuators, B, 2010, 145, 104-109.

17 G. Wang, X. Tan, Q. Zhou, Y. Liu, M. Wang and L. Yang, Sens. Actuators, B, 2014, 190, 730-736.

18 Y. Wang, J. Jin, C. Yuan, F. Zhang, L. Ma, D. Qin, D. Shan and X. Lu, Analyst, 2015, 140, 560-566.
19 H. Wang, Y. Su, H. Kim, D. Yong, L. Wang and X. Han, Chin. J. Chem., 2015, 33, 1135-1139.

20 N. K. Mogha, V. Sahu, M. Sharma, R. K. Sharma and D. T. Masram, Mater. Des., 2016, 111, 312-320.

21 X. Tian, L. Liu, Y. Li, C. Yang, Z. Zhou, Y. Nie and Y. Wang, Sens. Actuators, B, 2018, 256, 135-142.

22 J. M. George, A. Antony and B. J. M. A. Mathew, Microchim. Acta, 2018, 185, 358.

23 J. Gong, X. Miao, H. Wan and D. Song, Sens. Actuators, B, 2012, 162, 341-347.

24 M. Miller and S. Jong, in Developments in crop science, Elsevier, 1987, vol. 10, pp. 421-426.

25 P. Cheng, S. Gao, P. Zang, X. Yang, Y. Bai, H. Xu, Z. Liu and Z. Lei, Carbon, 2015, 93, 315-324.

26 W. S. Hummers Jr. and R. E. Offeman, J. Am. Chem. Soc., 1958, 80, 1339.

27 A. Singh and U. T. Nakate, Sci. World J., 2014, 2014, 349457.

28 R. D. Robinson, J. Tang, M. L. Steigerwald, L. E. Brus and I. P. Herman, Phys. Rev. B: Condens. Matter Mater. Phys., 2005, 71, 115408.

29 D. Padovini, D. Pontes, C. Dalmaschio, F. Pontes and E. Longo, $R S C A d v ., 2014,4,38484-38490$.

30 G. Liu and Y. Lin, Anal. Chem., 2005, 77, 5894-5901.

31 X. Yue, P. Han, W. Zhu, J. Wang and L. Zhang, RSC Adv., 2016, 6, 58771-58779.

32 J.-C. Ma and W.-D. Zhang, Microchim. Acta, 2011, 175, 309314.

33 D. Pan, S. Ma, X. Bo and L. Guo, Microchim. Acta, 2011, 173, 215-221.

34 S. Xia, J. Zhang and C. Li, Anal. Bioanal. Chem., 2010, 396, 697-705.

35 S. Yang, S. Luo, C. Liu and W. Wei, Colloids Surf., B, 2012, 96, 75-79.

36 D. Du, J. Liu, X. Zhang, X. Cui and Y. Lin, J. Mater. Chem., 2011, 21, 8032-8037.

37 S. Anandhakumar, K. Dhanalakshmi and J. Mathiyarasu, Electrochem. Commun., 2014, 38, 15-18.

38 Q. Zhang, Q. Du, T. Jiao, B. Pan, Z. Zhang, Q. Sun, S. Wang, T. Wang and F. J. C. e. j. Gao, Chem. Eng. J., 2013, 221, 315321. 